\title{
Intravesicle Bacillus Calmette-Guérin (BCG) Treatment for Non-Muscle Invasive Bladder Cancer (NMIBC) in the Transplanted Patients Population: A Systematic Review of the World Literature
}

\author{
Omer A Raheem ${ }^{1}$, Ehab A Eltahawy ${ }^{2}$, Rodney Davis ${ }^{2}$ and Mohamed H. Kamel ${ }^{\star 2}$ \\ ${ }^{1}$ Department of Urology, University of California, USA \\ ${ }^{2}$ Departments of Urology, University of Arkansas for Medical Sciences, USA
}

*Corresponding author: Mohamed H. Kamel, Associate Professor, Department of Urology, University of Arkansas for Medical Sciences, USA, Tel: 501-686-6916; Fax: 501-603-1422; E-mail: mkamel@uams.edu

Rec date: Nov 21, 2014, Acc date: Dec 15, 2014, Pub date: Dec 17, 2014

Copyright: (c) 2014 Raheem OA, et al. This is an open-access article distributed under the terms of the Creative Commons Attribution License, which permits unrestricted use, distribution, and reproduction in any medium, provided the original author and source are credited.

\begin{abstract}
Intravesicle Bacille Calmette-Guerin (BCG) is generally considered to be contraindicated in the immunosuppressed or compromised patients with bladder cancer (BC) because of ineffectiveness or partial toxicities. Therefore, there is little experience with BCG in individual with impaired immune system and this can be challenging to practicing urologists. We sought to review the current available evidence of utilizing intravesicle BCG for BC in patients with solid organ transplantation and commented on its current status.
\end{abstract}

Keywords: Bacillus calmette-guerin; Non muscle invasive bladder cancer; Transplanted patients

\section{Introduction}

Bladder cancer (BC) is the $8^{\text {th }}$ leading cause of cancer death in men in the United States (US). It is estimated that 74,690 patients will be diagnosed with $\mathrm{BC}$ in the US during the year 2014 [1]. In general, the non-metastatic BC is defined as a spectrum of bladder disease and traditionally classified into two broad categories: non-muscle invasive bladder cancer (NMIBC) and muscle invasive bladder cancer (MIBC). NMIBC is a bladder cancer that is limited to the bladder mucosa or lamina propria of the bladder wall. The distinction between NMIBC and MIBC is critical. NMIBC constitutes majority of BC (80\%) and can usually be treated with transurethral resection of bladder tumor (TURBT) and concomitant and/or subsequent administration of intravesicle treatments such as immunotherapy of Bacillus CalmetteGuérin (BCG) or chemotherapy of Mitomycin-C (MMC). Although $20 \%$ of BC cases presents with MIBC, these patients typically undergo more extensive local radical therapy with radical cystectomy and urine diversion or radiotherapy [2]. Recent contemporary data have shown that 5 years overall survival of NMIBC is $96 \%$ compared to $70 \%$ for the MIBC [1]

It is well established that solid organ transplantation is significantly associated with increased risk of BC. Buzzeo et al. examined the predicted risk of developing $\mathrm{BC}$ in the renal transplant recipients using the University of Wisconsin renal transplant database. Buzzeo's result demonstrated that the relative risk of developing $\mathrm{BC}$ in the renal transplant patients is 3.31 higher than the general population risk [3]. Ehdaie et al. examined the Surveillance, Epidemiology, and End Results (SEER) database defining the $\mathrm{BC}$ characteristics in the renal transplant patients' population, compared to all patients with $\mathrm{BC}$ included in the dataset [4]. This study highlights the patients with renal transplant and End-Stage Renal Disease (ESRD) presenting with $\mathrm{BC}$ tended to be younger, more likely to present with higher $\mathrm{BC}$ grade and stage [4]. Furthermore, BC was more likely to occur in the first 4 years post renal transplantation [4].

\section{Methods}

A detailed, comprehensive literature review was performed to identify all published peer-reviewed articles which describe intravesicle BCG treatment for $\mathrm{BC}$ and transplanted patients in the urological literature. The search was conducted through MEDLINE $^{\oplus}$ database, the Cochrane Library ${ }^{\oplus}$ Central Search, and the Web of Science. Initial search terms were BCG for BC and transplanted patients. Search results were screened for appropriate studies with particular emphasis placed on clinical and experimental studies as well as review articles. Articles referenced were screened to maximize review and inclusion of pertinent data. While English language text was not a specific search parameter, only English language publications were considered. All relevant studies collected were carefully examined to extract relevant data pertained to intravesicle BCG and transplanted patients.

\section{Evidence Synthesis for this Systematic Review}

Once it's first introduced, BCG vaccine is a live attenuated vaccine that was initially used as an intravesicle treatment for NMIBC originally described by Morales in 1976 [5]. Intravesicle BCG is considered an immunotherapy for treatment of BC. The mechanism of action involves binding to fibronectin in the bladder wall. This binding stimulates the production of several cytokines with strong antitumor affect including granulocyte macrophage colony stimulating factor (GM-CSF), tumor necrosis factors (TNF) $\alpha$, interferon (IF) $\gamma$ and multiple Interleukins (IL) in both the bladder wall and urine. A mechanism involving activation of T-helper response is evidenced by an increase in IF- $\mathrm{\gamma}$ and IL-2 and IL-12 [6]. Intravesicle BCG is considered the most effective adjuvant treatment for NMIBC following transurethral resection of bladder tumors [7]. It is effective in reducing recurrence and progression of NMIBC by $40 \%$ and $25 \%$ 
respectively than transurethral resection of bladder tumors alone $[7,8]$. BCG is normally administered as an induction course of 6 weeks of intravesicle treatments followed by maintenance protocol. The rationale behind the initial intense course of 6 weeks BCG is to induce a robust local immune therapy in the bladder, while maintenance therapy intermittently provides boost to that immune response to keep it up-regulated. The frequency and duration of maintenance protocol is controversial but it is usually for 2-3 years following the induction course [9].

Intravesicle $\mathrm{BCG}$ is associated with higher complications rate compared to intravesicle chemotherapy as Epirubicin or MMC. However serious side effects occur in less than 5\% [10]. Side effects are usually classifies into local side effects including hematuria (most common), frequency, severe dysuria and chemical or bacterial cystitis. Systemic toxicity is mainly due to absorption of the drug through the bladder wall and includes fever, skin rash, generalized malaise, systemic infection and septicemia. The rate of drop out from treatment secondary to side effects is reported to be $20 \%$ mostly due to BCG induced cystitis [10]. Measures to reduce the risk of BCG side effects include administration of the drug 2-3 weeks after TURBT, in the absence of gross hematuria or active urinary tract infection and most important; use of gentle catheterization technique. These measures aim to reduce the risk of intravasation of the drug in the blood stream. Absolute contraindications to Intravesicle BCG treatment includes Immunocompromised or immunosuppressed individuals, traumatic catheterization, gross hematuria, previous history of BCG sepsis and active urinary tract infections [7]. Notably, asymptomatic bacteruia is not a contraindication for intravesical BCG [11].

\section{Current Controversies on Using Intravesicle BCG in the Transplanted Patients}

Solid organ transplanted patients with NMIBC candidates for intravesicle BCG often present a challenge to practicing urologists. There are concerns that these patients, because of their immune status, may not be able to mount a sufficient immune response following intravesicle BCG administration to eradicate BC. Moreover, there is a concern for a potential risk of developing bacterial or BCG sepsis in these immunocompromised patients [12]. Novel immunosuppressive agents used post-transplantation are specifically inhibitors to IL production, an important mechanism through which intravesicle BCG exerts its antitumor effect [13]. In addition, steroids remain a main stay drug in post-transplant immunosuppressive regimens. Importantly, it is well known that transplant patients are at increased risk of bacterial and viral infections due to immunosuppressed status. The risk of Tuberculosis infection in the solid organ transplant recipients is 20-74 times higher than the general population and the treatment is associated with significant morbidity and mortality [14]. Historical published reports described an increased risk of complications following intralesional injection of BCG vaccine in cancer patients $[15,16]$. Although, these concerns do exist, there is limited data to support that intravesicle BCG treatment for NMIBC is unsafe or ineffective in the solid organ transplant patients. More recently, published data have validated the safety and efficacy of intravesicle BCG in the transplant and immunocompromised patients. However, these reports have inherited flaws owing to the small number of patients and retrospective designs.

\section{Current Status of Intravesicle BCG in the Transplanted Patients}

Palou et al. described the first use intravesicle BCG in the transplant patients on 3 patients. Two patients remained recurrence free at 17 and 60 months follow up and one patient developed recurrent Carcinoma In-Situ (CIS) managed by radical cystectomy and urine diversion. Pathology report for the last patient showed no residual tumor in the bladder (T0). None of the 3 patients developed a complication secondary to the use of intravesicle BCG [17]. In another study, 4 patients with solid organ transplant and NMIBC (3 patients with CIS and 1 patient with T1 disease) were treated with Intravesicle BCG. All patients at the time of intravesicle BCG administration were receiving Tacrolimus, 2 in addition were receiving prednisone and one receiving Mycophenolate Mofetil. Three of the 4 patients developed local recurrences in the bladder successfully managed by further intravesicle BCG or MMC and at a mean of 51 months all 4 patients are alive and recurrence free [18]. Recent study by Herr and Dalbagni reported on the outcome of induction intravesicle BCG in 45 immunocompromised patients treated at the Memorial Sloan Kattering Cancer Center (MSKCC). Of this cohort, 12 patients were transplant patients, 23 were receiving systemic chemotherapy for unrelated cancers and 10 were taking steroids for autoimmune disorders. The results of this study were sobering. In all patients BCG was well tolerated and none of the patients developed bacterial or BCG sepsis. There was no decline in graft function attributed to BCG. At 6 months follow up, 9 out of the12 transplant patients, all 23 patients with unrelated cancers and nine of the 10 patients with autoimmune disease had complete response to one or 2 cycles of BCG. At a median follow up of 40 months, only 1 of the 12 transplant patients remained recurrence free. The median recurrence free survival in the transplant group was 17 months. In terms of progression, the median progression free survival in the transplant group was 40 months. Six of the 12 transplant patients eventually progressed. Two developed metastasis with local relapse and eventually died, 3 were managed by radical cystectomy and at the time of the report were alive and 1 patient developed recurrence of urothelial carcinoma in the transplant kidney and was managed by endoscopic surgery. The response to BCG in the groups of patients with autoimmune disease and unrelated cancers fared better than the transplant patients group. The authors concluded that the response rate for the 45 patients as a whole compared favorably with their previous reports on the outcome of intravesicle BCG in the non-immunocompromised patients with bladder cancer $[19,20]$.

\section{Conclusions}

Although recent data have shown safety and efficacy of utilizing intravesicle BCG in treatment of NMIBC in the immunosuppressed patients, including transplants, there remains significant lack strong clinical evidence to supporting its widespread usage among transplanted patients. Additionally, intravesicle BCG appears to be well tolerated and effective against immunosuppressed patients with high-risk NMIBC that can be treated successfully with intravesicle BCG and may achieve results similar to non-immunosuppressed patients. It is equally important to report that urologists should interpret these current results with caution as intravesicle BCG can be associated with serious adverse effects particularly in the transplanted population. Once given, these patients should be closely monitored for any BCG side effects and/or BCG failure or recurrent BC with the view of prompt radical cystectomy. Future research should be directed to 
Citation: Raheem OA, Eltahawy EA, Davis R, Kamel MH (2015) Intravesicle Bacillus Calmette-Guérin (BCG) Treatment for Non-Muscle Invasive Bladder Cancer (NMIBC) in the Transplanted Patients Population: A Systematic Review of the World Literature . J Transplant Technol Res 5: 144. doi:10.4172/2161-0991.1000144

Page 3 of 3

enhance our molecular immunologic knowledge and interactions in these specific BC cases and how to avoid serious adverse effects of BCG therapies in the face of organ transplantation. In addition, large randomized clinical trials are pre-requisite to validate the safety and efficacy of intravesicle BCG for BC treatment in the transplanted patients where patients' safety and outcomes are end-points.

\section{References}

1. Cancer Facts and Figures (2014) Statistics adapted from the American Cancer Society's publication.

2. Berglund RK and Herr H (2012) Surgery for Bladder cancer: CampbellWalsh Urology. Elsevier-Saunders.

3. Buzzeo BD, Heisey DM, Messing EM (1997) Bladder cancer in renal transplant recipients. See comment in PubMed Commons below Urology 50: 525-528.

4. Ehdaie B, Stukenborg GJ, Theodorescu D (2009) Renal transplant recipients and patients with end stage renal disease present with more advanced bladder cancer. See comment in PubMed Commons below J Urol 182: 1482-1487.

5. Morales A, Eidinger D, Bruce AW (1976) Intracavitary Bacillus Calmette-Guerin in the treatment of superficial bladder tumors. J Urol 116: $180-183$

6. Böhle A, Brandau S (2003) Immune mechanisms in bacillus CalmetteGuerin immunotherapy for superficial bladder cancer. See comment in PubMed Commons below J Urol 170: 964-969.

7. Jones SJ, Larchain WA (2012) Non-Muscle-Invasive Bladder Cancer (Ta, T1, and CIS): Campbell-Walsh Urology. Elsevier-Saunders.

8. Herr HW, Schwalb DM, Zhang ZF, Sogani PC, Fair WR, et al. (1995) Intravesical bacillus Calmette-Guérin therapy prevents tumor progression and death from superficial bladder cancer: ten-year followup of a prospective randomized trial. J Clin Oncol 13: 1404-1408.

9. O'Donnell MA1 (2005) Practical applications of intravesical chemotherapy and immunotherapy in high-risk patients with superficial bladder cancer. See comment in PubMed Commons below Urol Clin North Am 32: 121-131.

10. Sylvester RJ, van der Meijden AP, Oosterlinck W, Hoeltl W, Bono AV, et al. (2003) The side effects of Bacillus Calmette-Guerin in the treatment of
Ta T1 bladder cancer do not predict its efficacy: results from a European Organisation for Research and Treatment of Cancer Genito-Urinary Group Phase III Trial. Eur Urol 44: 423-428.

11. Herr HW (2012) Intravesical bacillus Calmette-Guérin outcomes in patients with bladder cancer and asymptomatic bacteriuria. See comment in PubMed Commons below J Urol 187: 435-437.

12. Babjuk M, Oosterlinck W, Sylvester R, Kaasinen E, Böhle A, et al. (2011) EAU guidelines on non-muscle-invasive urothelial carcinoma of the bladder, the 2011 update. Eur Urol 59: 997-1008.

13. Balbontin F, Kiberd B, Fraser A, Kiberd M, Lawen J (2005) Basiliximab lowers the cyclosporine therapeutic threshold in the early post-kidney transplant period. See comment in PubMed Commons below Clin Transplant 19: 225-229.

14. García-Goez JF, Linares L, Benito N, Cervera C, Cofán F, et al. (2009) Tuberculosis in solid organ transplant recipients at a tertiary hospital in the last 20 years in Barcelona, Spain. Transplant Proc 41: 2268-2270.

15. Sparks FC, Silverstein MJ, Hunt JS, Haskell CM, Pilch YH, et al. (1973) Complications of BCG immunotherapy in patients with cancer. See comment in PubMed Commons below N Engl J Med 289: 827-830.

16. McKhann CF, Hendrickson CG, Spitler LE, Gunnarsson A, Banerjee D, et al. (1975) Immunotherapy of melanoma with BCG: two fatalities following intralesional injection. See comment in PubMed Commons below Cancer 35: 514-520.

17. Palou J, Angerri O, Segarra J, Caparrós J, Guirado L, et al. (2003) Intravesical bacillus Calmette-Guèrin for the treatment of superficial bladder cancer in renal transplant patients. See comment in PubMed Commons below Transplantation 76: 1514-1516.

18. Tomaszewski JJ, Larson JA, Smaldone MC, Hayn MH, Jackman SV (2011) Management of Bladder Cancer following Solid Organ Transplantation. See comment in PubMed Commons below Adv Urol 2011: 256985.

19. Herr HW, Dalbagni G (2013) Intravesical bacille Calmette-Guérin (BCG) in immunologically compromised patients with bladder cancer. See comment in PubMed Commons below BJU Int 111: 984-987.

20. Herr HW, Dalbagni G, Donat SM (2011) Bacillus Calmette-Guérin without maintenance therapy for high-risk non-muscle-invasive bladder cancer. See comment in PubMed Commons below Eur Urol 60: 32-36. 